\title{
Results of erosion processes of brown mountainous-steppe soils of the southeastern part of the Small - Caucasus
}

\author{
RAE ZH Aliyev* \\ Institute of Erosion and Irrigation NAS of Azerbaijan, Azerbaijan
}

Received: September 15, 2017; Published: September 26, 2017

*Corresponding author: RAE ZH Aliyev, Institute of Erosion and Irrigation NAS of Azerbaijan, Azerbaijan, Email: zakirakademik@mail.ru

\section{Introduction}

Brown mountainous-steppe soils arranged in the form of spots in separate forest edges and meadows within the zone of brown mountainous- forest soils. The lower boundary of these soils coincides with the upper boundary of gray-brown mountainous soils. The problem of steppification of forest soils was widely covered in the writings of the V.V. Akimsev [1], S.A. Zakharov 1937, S.V.Zonn [2] and other researchers. Information on Azerbaijan, on the processes of steppification of mountain-forest soils is available in the works of G.A Aliyev [3], ME. Salayev 1966, K.A. Alekberov [4], Sh.H Hasanov [5], and others. In these researches, it is shown that the process of soil steppification occurs under the influence of human economic activity.

G.A.Aliyev [3] notes that steppification of forest soils is under the influence of human activity. The harvesting and piling of the forest started, mainly from the lower boundary of the forest zone. This is natural, because in these territories, climatic conditions contribute to the development of agricultural crops, the relief of the terrain is convenient both for the agricultural use that made free from forest massive. Regarding with transportation of wood, Sh.H. Hasanov [5] points out that by releasing more accessible and convenient territories, especially at the lower boundary of the forest, for their economic needs, a person thereby contributes to a change of forest biocoenosis by a more xerophytic vegetation formation, represented by oak, Christ's-thorn, hawthorn, blackberry and others.

Brown mountainous steppe soils are mainly used for planting of agricultural crops. Agriculture here is dry, a part of the plowed land on the slopes, as a result of the absence of soil-protective agrotechnical methods of soil treatment, and in connection with the lack of anti-erosion measures, is leaded to a condition unsuitable for agricultural use. The described soils occupy 25175,0 hectares or $35.61 \%$ of the total area of the researched territory. Of these, 9565.0 hectares or $37.99 \%$ are not eroded, 7345,0 hectares or $29.18 \%$-are slightly eroded, 5615,0 hectares or $22.30 \%$ are medium-eroded, 2650,0 hectares or $10,53 \%$ strongly eroded.

In order to characterize the morphological parameters of these soils, a description is provided in the section No. 9, which lies 0.8 $\mathrm{km}$ to the west from the village of Aghjakend, on the northern slope with steepness 13 . The plot is used under haymaking. A $0-19 \mathrm{~cm}-$ brown, darkish, light-clayish, big-scale nut proportion, dense, very much roots and rootlets, the channels of earthworms, wet, clear transition, boiled from HCL.

a) A 19-48cm -brown, heavy loamy, lumpy, dense, less roots and rootlets, the channels of earthworms, wet, the transition is gradual, boiled from HCL.

b) C $48-74 \mathrm{~cm}$ - light- gray-brown, clayish, fine-grained, dense, there are roots and fragments of rocks, wet, transition is sharp, boiled from HCL.

c) C $74-105 \mathrm{~cm}$ - white, clayish, structure-less, and dense, many fragments of rocks, wet, boiled strongly from HCL.

d) The humus horizon is shortened and their morphological structure changes in the eroded ponds of the described soils. We give a description of section No. 15, laid $1.5 \mathrm{~km}$ north-west from the village of Dolanlar, on the northeastern slope with a steepness of 17 to characterize their highly washed differences. The site is used for planting pastures.

e) B0-24cm-gray-brown, medium loamy, weakly lumpy, little roots and rootlets, channels of earthworms, fragments of rocks, wet, clear transition, boiled strongly from HCL.

f) BC 24-45cm-gray-brown, heavy loamy, structureless, dense, very few roots, fragments of rocks, wet, boiled roughly from HCL.

According to the above stated description of the morphological structure, the coloration of mountain - brown steppe soils gradually becomes lighter along the profile. There is significant claying in the middle and lower parts of the profile. Table 1 presents the results of a mechanical analysis of mountainous brown steppe soils. The volume of physical clay is $63.40 \%$, the silt fraction is $28.96 \%$, declines up to $40,60 \%$, and $18,00 \%$ in the strongly eroded specimens of the upper horizon of their unwashed examples. Erosion processes also affect the chemical composition and the content of nutrient 
elements. The content of humus is $4.83 \%$, total nitrogen $0.29 \%, \quad 0.09 \%$, and $0.07 \%$ (Table 2 ) in strongly eroded specimens (Table total phosphorus $0.22 \%$ in the upper horizon of unwashed brown $1-3$ ).

mountainous steppe soils; it declines correspondingly up to $1.43 \%$;

Table 1 : Mechanical composition of brown steppe mountainous soils (on \% of complete dry soil).

\begin{tabular}{|c|c|c|c|c|c|c|c|c|c|c|c|}
\hline \multirow[b]{2}{*}{ Cut No } & \multirow{2}{*}{$\begin{array}{l}\text { Washing } \\
\text { degree }\end{array}$} & \multirow{2}{*}{$\begin{array}{c}\text { Genetic } \\
\text { zones }\end{array}$} & \multirow{2}{*}{$\begin{array}{c}\text { Depth of } \\
\text { sampling, } \\
\text { cm }\end{array}$} & \multirow{2}{*}{$\begin{array}{c}\text { Absorbed } \\
\text { moisture, } \\
\%\end{array}$} & \multicolumn{7}{|c|}{ Proportions of bodies, $\mathrm{mm}$} \\
\hline & & & & & $1-0,25$ & $0,25-0,05$ & $0,05-0,01$ & $\begin{array}{l}0,01- \\
0,005\end{array}$ & $\begin{array}{c}0,005- \\
0,001\end{array}$ & $<0,001$ & $<0,01$ \\
\hline \multirow{4}{*}{19} & \multirow{4}{*}{ Unwashed } & A1 & $0-19$ & 6,14 & 9,40 & 14,20 & 13,00 & 3,00 & 31,44 & 28,96 & 63,40 \\
\hline & & A2 & $19-48$ & 6,10 & 2,56 & 11,64 & 26,00 & 6,40 & 23,64 & 29.76 & 59.80 \\
\hline & & $\mathrm{B}$ & $48-74$ & 6,21 & 1,34 & 17,26 & 9,36 & 15,24 & 19,68 & 37,12 & 72,04 \\
\hline & & $\mathrm{C}$ & $74-105$ & 5,66 & 2.93 & 16,67 & 9,24 & 9,16 & 53,40 & 8,60 & 71,16 \\
\hline \multirow{3}{*}{8} & \multirow{3}{*}{$\begin{array}{l}\text { Weakly } \\
\text { eroded }\end{array}$} & A & $0-20$ & 5,67 & 14,50 & 12,70 & 16,80 & 15,20 & 24,80 & 16,00 & 56,00 \\
\hline & & $\mathrm{B}$ & $20-37$ & 6,22 & 11,50 & 12,10 & 20,80 & 7,60 & 18,00 & 20,00 & 55,60 \\
\hline & & $\mathrm{BC}$ & $37-78$ & 5,74 & 23,30 & 17,50 & 24,40 & 6,80 & 21.04 & 6,96 & 34.80 \\
\hline \multirow{3}{*}{48} & \multirow{3}{*}{$\begin{array}{l}\text { Moderately } \\
\text { eroded }\end{array}$} & $\mathrm{AB}$ & $0-29$ & 5,99 & 3,30 & 10,70 & 40,00 & 10,20 & 5,80 & 30,00 & 46,00 \\
\hline & & B & $29-58$ & 6,25 & 4,34 & 6,22 & 20,64 & 14,80 & 18.40 & 35,60 & 68,80 \\
\hline & & $\mathrm{C}$ & $58-72$ & 5,79 & 2,55 & 15.45 & 17,12 & 15,88 & 22,60 & 26,40 & 64,88 \\
\hline \multirow{2}{*}{15} & \multirow{2}{*}{$\begin{array}{l}\text { Strongly } \\
\text { eroded }\end{array}$} & B & $0-24$ & 4,40 & 11,12 & 24,80 & 13,48 & 14,60 & 8,00 & 18,00 & 40,60 \\
\hline & & $\mathrm{BC}$ & $24-45$ & 6,32 & 11,36 & 26,64 & 13,00 & 15,80 & 20,20 & 13,00 & 49,00 \\
\hline
\end{tabular}

Table 2: Some chemical properties of brown steppe mountainous soils.

\begin{tabular}{|c|c|c|c|c|c|c|c|c|c|c|c|c|c|c|}
\hline \multirow{2}{*}{$\begin{array}{l}\text { Cut } \\
\text { No }\end{array}$} & \multirow{2}{*}{$\begin{array}{l}\text { Erosion } \\
\text { degree }\end{array}$} & \multirow{2}{*}{$\begin{array}{l}\text { Genetic } \\
\text { zones }\end{array}$} & \multirow{2}{*}{$\begin{array}{c}\text { Depth of } \\
\text { sampling, } \\
\text { cm }\end{array}$} & \multirow{2}{*}{$\begin{array}{c}\text { Humus } \\
\qquad \%\end{array}$} & \multicolumn{2}{|c|}{ Total, \% } & \multirow{2}{*}{$\begin{array}{c}\text { Absorbed } \\
\text { basis in } \\
100 \text { gr. } \\
\text { soil with } \\
\text { mg/ekv } \\
\text { Ca }\end{array}$} & \multirow[b]{2}{*}{ Mg } & \multirow[b]{2}{*}{ Total } & \multirow{2}{*}{$\begin{array}{c}\text { Nutrients } \\
\text { mg in } \\
\text { each } 1 \mathrm{~kg} \\
\text { of soil }\end{array}$} & \multirow[b]{2}{*}{$\kappa_{2} \mathbf{O}$} & \multirow{2}{*}{$\begin{array}{c}\mathrm{pH} \\
\text { water }\end{array}$} & \multirow{2}{*}{$\begin{array}{c}\mathrm{Co}_{2} \\
\text { carbon } \\
\text { atov, }\end{array}$} & \multirow{2}{*}{$\begin{array}{l}\mathrm{CaCO}_{3} \\
\mathrm{Co}_{2} \%\end{array}$} \\
\hline & & & & & Nitrogen & Phosphorus & & & & & & & & \\
\hline \multirow{4}{*}{19} & \multirow{4}{*}{ Unwashed } & A1 & $0-19$ & 4,83 & 0,29 & 0,22 & 35,85 & 4,41 & 40,26 & 28,8 & 450,6 & 7,5 & 0,98 & 2,22 \\
\hline & & $\mathrm{A} 2$ & $19-48$ & 3,38 & 0,27 & 0,16 & 29,14 & 3,76 & 32,90 & 17,0 & 114,5 & 7,7 & 1,61 & 3,65 \\
\hline & & B & $48-74$ & 1,09 & 0,05 & 0,12 & 27,26 & 5,64 & 32,90 & 10,0 & 186,8 & 7,8 & 1.37 & 3,11 \\
\hline & & $\mathrm{C}$ & 74-105 & 0,72 & 0,04 & 0,06 & 23,03 & 2,47 & 25,50 & 5,8 & 132,5 & 7,9 & 3.1 & 7,04 \\
\hline \multirow{3}{*}{8} & \multirow{3}{*}{$\begin{array}{l}\text { Weakly } \\
\text { eroded }\end{array}$} & $\mathrm{A}$ & $0-20$ & 3,55 & 0,28 & 0,18 & 26,32 & 3,29 & 29,61 & 16,0 & 253,0 & 7,8 & 1,34 & 3,04 \\
\hline & & $\mathrm{B}$ & $20-37$ & 2,83 & 0,20 & 0,14 & 27,26 & 2,35 & 29,61 & 9,2 & 180,7 & 7,8 & 3,92 & 8,90 \\
\hline & & $\mathrm{BC}$ & $37-78$ & 0,78 & 0,07 & 0,10 & 11,28 & 3,76 & 15,04 & 8,0 & 114,5 & 7,9 & 7,06 & 16,03 \\
\hline \multirow{3}{*}{48} & \multirow{3}{*}{$\begin{array}{c}\text { Moderately } \\
\text { eroded }\end{array}$} & $\mathrm{AB}$ & $0-29$ & 3,47 & 0,20 & 0,12 & 21,15 & 4,23 & 25,38 & 13,6 & 192,8 & 7.9 & 3,95 & 8,97 \\
\hline & & $\mathrm{B}$ & $29-58$ & 1,79 & 0,10 & 0,08 & 22,09 & 1,41 & 23,50 & 11,8 & 108,4 & 8,0 & 4,84 & 10,99 \\
\hline & & $\mathrm{C}$ & $58-72$ & 1,67 & 0,10 & 0,06 & 14,74 & 5,17 & 19,91 & 11,6 & 78,3 & 8,1 & 5,83 & 13,23 \\
\hline \multirow{2}{*}{15} & \multirow{2}{*}{$\begin{array}{l}\text { Strongly } \\
\text { eroded }\end{array}$} & $\mathrm{B}$ & $0-24$ & 1,43 & 0,09 & 0,07 & 18,80 & 3,41 & 22,21 & 13,8 & 150,6 & 8,1 & 3,89 & 8,83 \\
\hline & & $\mathrm{BC}$ & $24-45$ & 0,31 & 0,01 & 0,05 & 17,86 & 3,76 & 21,62 & 10,0 & 84,3 & 8.2 & 7,91 & 17,96 \\
\hline
\end{tabular}

$\underline{\text { Table } 3}$ : SStructure (numerator) and aggregate composition (denominator) of steppe mountainous Soils.

\begin{tabular}{|c|c|c|c|c|c|c|c|c|c|c|c|c|}
\hline \multirow[b]{2}{*}{ Cut No } & \multirow{2}{*}{$\begin{array}{l}\text { Erosion } \\
\text { degree }\end{array}$} & \multirow{2}{*}{$\begin{array}{l}\text { Genetic } \\
\text { zones }\end{array}$} & \multirow{2}{*}{$\begin{array}{l}\text { Depth of } \\
\text { sampling, } \\
\text { cm }\end{array}$} & \multicolumn{9}{|c|}{ Particles with mm } \\
\hline & & & & $>7$ & 5-Jul & 3-May & 1-Mar & $1-0,5$ & $\begin{array}{l}0,5- \\
0,25\end{array}$ & $<0,25$ & $>1$ & $\%$ \\
\hline \multirow{7}{*}{19} & \multirow{7}{*}{ Unwashed } & $\mathrm{A} 1$ & $0-19$ & $21,814,2$ & 92 & $30,225,7$ & $32,728,9$ & 2.5 & 2.1 & 1.5 & $93,981.3$ & 2,2 \\
\hline & & & & & 12,5 & & & 11,4 & 2,2 & 2,9 & & \\
\hline & & A2 & $19-48$ & $21,510,8$ & $21,98,9$ & $26,216,4$ & $26,320,7$ & 1.8 & 1.1 & 1.2 & $95,956,8$ & 3,8 \\
\hline & & & & & & & & 8,4 & 1,9 & 29,1 & & \\
\hline & & B & $48-74$ & $17,610,2$ & $26,213,1$ & $28,714,2$ & $22,118,8$ & $0.710,3$ & 1 & 3.7 & $94,656,3$ & 5,3 \\
\hline & & & & & & & & & 14,5 & 14,6 & & \\
\hline & & $\mathrm{C}$ & $74-105$ & $14,156,1$ & $16,610,9$ & $13.58,2$ & $32,323,2$ & $6.6,6,8$ & $7.5,15,4$ & $9.4,26,6$ & $76.548,4$ & 2,8 \\
\hline
\end{tabular}




\begin{tabular}{|c|c|c|c|c|c|c|c|c|c|c|c|c|}
\hline \multirow{3}{*}{8} & \multirow{3}{*}{$\begin{array}{l}\text { Weakly } \\
\text { eroded }\end{array}$} & A & $0-20$ & $15,93,8$ & $28,710,6$ & $26,914,8$ & $17,321,0$ & $4.3,11,4$ & $4,3,4$ & $2.918,2$ & $88,850,2$ & 16.8 \\
\hline & & B & $20-37$ & $14,88,4$ & $15,211,2$ & $32,217,6$ & $29,310,8$ & $1.94,9$ & $2.6,2,2$ & $4,0,34,0$ & $91,548,0$ & 10,9 \\
\hline & & $\mathrm{BC}$ & $37-78$ & $9.4,6,1$ & $23,75,5$ & $19,54,8$ & $23,817,6$ & $6.511 ., 4$ & $7.27,2$ & $9.9,40,1$ & $76.434,0$ & 7,3 \\
\hline \multirow{2}{*}{48} & \multirow{2}{*}{$\begin{array}{l}\text { Moderately } \\
\text { eroded }\end{array}$} & $\mathrm{AB}$ & $0-29$ & $17,68,5$ & $7.4,6,2$ & $9.38,4$ & $40,226,7$ & $8.1,10,8$ & $9.8,4,9$ & $7.6,23,9$ & $74,549,7$ & 10,7 \\
\hline & & $\mathrm{B}$ & $29-58$ & 922,6 & $17,14,9$ & $31,910,2$ & $25,422.4$ & $2.8,3,6$ & $4.4,7,7$ & $9.2,34,3$ & $83,640,1$ & 14,3 \\
\hline \multirow{2}{*}{15} & \multirow{2}{*}{$\begin{array}{l}\text { Strongly } \\
\text { eroded }\end{array}$} & B & $0-24$ & 5.1 & $23,20,8$ & $13,314,2$ & $19,814,8$ & $14.1,1,0$ & $12,26,2$ & $\begin{array}{l}12,3 \\
38,5\end{array}$ & $61.429,8$ & 24.5 \\
\hline & & $\mathrm{BC}$ & $24-45$ & 4.7 & 20,8 & $12,92,9$ & $18,019,5$ & $18,9,2,3$ & $11.4,4.8$ & $\begin{array}{l}13,3 \\
40,5\end{array}$ & $\begin{array}{l}56.4 \\
22,4\end{array}$ & 30,0 \\
\hline
\end{tabular}

The absorption capacity $(\mathrm{Ca}+\mathrm{Mg})$ is $40.26 \mathrm{mg} / \mathrm{ekv}$ in the upper horizon of unwashed soils, $25.38 \mathrm{mg} / \mathrm{ekv}$ of mediumeroded soils, and $22.21 \mathrm{mg} / \mathrm{ekv}$ in each 100 gr of highly eroded soils. The amount of nutrients in brown mountainous steppe soils significantly decreases, as the degree of erosion increases. Thus, the content of assimilable phosphorus is $28.8 \mathrm{mg} / \mathrm{kg}$, the exchangeable potassium $450.6 \mathrm{mg} / \mathrm{kg}$ in the upper horizon of their uneroded soils. The index of $\mathrm{pH}$ is 7.5-8.2. The amount of $\mathrm{CaCO}_{3}$ is $2.22-8.97 \%$ in the upper horizons of these soils, and $7.04-17.96 \%$ in the lower soils.

\section{References}

1. Akimtsev VV (1928) Soils of the Ganja region. "Materials on regionalization of Azerbaijan USSR" T.2 p. 3-105.

2. Zonn SV (1983) "Modern problems of genesis and geography of soils. M. Nauka p. 168.

3. Aliyev HA (1965) Brown forest soils (within the eastern part of the Greater Caucasus) Edition of AS of Azerb. USSR p. 112.

4. Alekberov KA (1961) Soil erosion in the Azerbaijan USSR and struggle with it (in Azerbaijani), Baku p. 220.

5. Hasanov Sh G (1978) Genetic features and soil classification of Southwest Azerbaijan, Baku, 1978, p. 220.

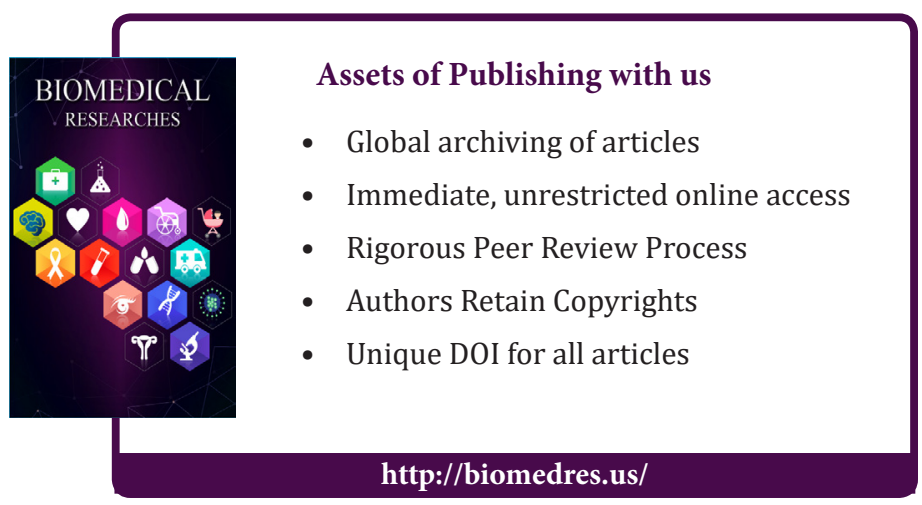

